\title{
Primary treatment and Recent survival trends in patients with Primary Diffuse Large B-Cell Lymphoma of Central Nervous System,1995-2016: a population-based SEER analysis
}

\section{Cui Chen}

First Affiliated Hospital of Sun Yat-sen University

\section{Peng Sun}

Sun Yat-Sen University Cancer Center

\section{Xiaoqing Sun}

Sun Yat-Sen University Cancer Center

\section{Shaoyong Chen}

Meizhou People's Hospital, Meizhou Hospital Affiliated to Sun Yat-sen University

\section{Hang Yang}

Sun Yat-Sen University Cancer Center

\section{Yu Wang}

Sun Yat-Sen University Cancer Center

Zhi-ming Li ( $D$ zhi_mingli@163.com )

Sun Yat-Sen University Cancer Center

\section{Research Article}

Keywords: Primary central nervous system lymphoma, Diffuse large B-cell lymphoma, SEER, Survival trends, Primary treatment

Posted Date: April 2nd, 2021

DOI: https://doi.org/10.21203/rs.3.rs-325917/v2

License: (9) This work is licensed under a Creative Commons Attribution 4.0 International License. Read Full License 


\section{Abstract}

This retrospective cohort study aimed to evaluate primary treatment and recent survival trends in patients with primary diffuse large B-cell lymphoma of CNS from 1995 to 2016.Using the SEER data, patients diagnosed with non-HIV associated PCNSL-DLBCL aged $\geqslant 18$ years between 1995 and 2016 were identified. The year of diagnosis was divided into the time periods-1(1995-2002), the time periods2(2003-2012) and the time periods-3(2013-2016). We used Chi-square tests, the Kaplan-Meier method, log-rank test and Cox regression model in the analysis. Overall, 3760 patients were included. Both the use of radiotherapy alone and the application of combined chemoradiotherapy decreased significantly, following the wider use of chemotherapy alone during 1995-2016. There was a significant improvement in PCNSL cancer-specific survival (period-1: 13 months vs period-2: 19 months vs period-3: 41 months, $P$ $<0.001)$. Survival of patients aged above 70 years did not change from the time period- 1 to the time period-2 $(P=0.101)$. However, there was an increase in CSS from the time period- 2 to the time period-3 in the elderly patients (period-2: 5 months vs period-3: 9 months, $P<0.001$ ). On multivariable analyses, diagnosed in the time period-3 was significantly and independently associated with better CSS (HR 0.577 , $95 \% \mathrm{Cl} 0.506-0.659)$.

Our analysis shows the use of radiotherapy in the treatment of PCNSL has waned over the study span. There was a significant improvement in CSS during 1995-2016, which reflected developments in treatment over time. The elderly patient population also gained a significant CSS benefit in the most recent period.

\section{Introduction}

Primary central nervous system lymphoma (PCNSL) is among the most aggressive variants of extranodal non-Hodgkin lymphoma that involves the brain, leptomeninges, eyes, or spinal cord without evidence of systemic involvement ${ }^{1}$. $90 \%-95 \%$ of PCNSLs are pathologically diagnosed as diffuse large B-cell lymphoma (DLBCL) ${ }^{1,2}$. Progress in PCNSL treatment have been gained in the past two decades ${ }^{3,4}$.

PCNSL tends to be highly sensitive to both radiotherapy and chemotherapy. Whole-brain radiotherapy (WBRT) was regarded as the upfront treatment for PCNSL in the early 1990s and achieved a median overall survival (OS) of approximately 1 year ${ }^{5}$. In the 1990s, the HDMTX-based combination chemotherapy followed by additional consolidative WBRT was regarded 'standard' treatment for PCNSL by many researchers. The approach resulted in high response rate of more than $90 \%$, median OS of $12-$ 60 months and 2-years OS of $60-85 \%$ in several prospective trials ${ }^{6-9}$. A non-cross-resistant conventional chemotherapy or high-dose chemotherapy with autologous stem cell transplantation showed good outcomes as options for consolidation therapy in some studies in the 2000s $\mathrm{s}^{10-13}$. One of the consolidation regimens was chosen according to balance the benefit and risk of each regimen in clinical practice since then. Based mainly on evidence of benefit in systemic CD20-positive lymphomas, rituximab is also commonly administered along with high dose methotrexate (HDMTX)-based chemotherapy for PCNSL from the 2000s ${ }^{14}$. Although the response rates to induction and consolidation 
therapy are high, almost half of these patients will relapse generally. Salvage chemotherapy regimens containing temozolomide, pemetrexed and topotecan, either in combination with others or as a single agent, have resulted in response rates of $14 \%-53 \%{ }^{15,16}$. Since 2013 , novel agents which are less toxic, lenalidomide and the Bruton's tyrosine kinase (BTK) inhibitor ibrutinib have shown activities in several clinical trials ${ }^{17-19}$. The efficacy of PD-1 checkpoint inhibitor for salvage therapy of PCNSL is also being considered $^{20}$.

The clinical efficacy of these new consolidation protocols and agents has been established in a series of clinical studies. However, the results from such studies may not reflect the actual clinical practice because of the stringent eligibility criteria for clinical trials. Population-based studies which based on the general patient population may complement prospective intervention studies. Therefore, we report the outcomes of a population-based SEER retrospective cohort study on primary treatment and survival trends among adult PCNSL patients between 1995 and 2016.

\section{Patients And Methods}

\subsection{Data source and patient selection}

The current retrospective cohort study relied on the SEER database (1975-2016), which samples $28 \%$ of the United States and publishes data of cancer incidence, treatment, and survival from population-based cancer registries ${ }^{21}$. In the SEER database, we focused on patients with PCNSL from the SEER-18 to conduct this analysis. The SEER-18 registry includes Atlanta, Detroit, Greater California, Greater Georgia, Hawaii, lowa, Kentucky, Los Angeles, New Mexico, New Jersey, Rural Georgia, states of Connecticut, San Francisco-Oakland, Seattle-Puget Sound, San Jose Monterey, the Alaska Native Tumor Registry, Louisiana, and Utah.

The SEER database classifies cancer histology and tomography by using the third edition of the International Classification of Disease-Oncology (ICD-0-3). PCNSL was defined by cancer diagnoses in anatomic locations of brain, spinal cord, leptomeninges, and other parts of the CNS (ICD-0-3 codes C70.0C72.9). PCNSL patients with diffuse large B-cell histology were identified in SEER by filtering the databases based on histology codes (9680, diffuse large B-cell lymphoma [DLBCL], NOS; 9684, malignant lymphoma, large B, diffuse, immunoblastic; 9688, T-cell histiocyte-rich large B-cell lymphoma).

For this study, we included patients with primary diffuse large B-Cell lymphoma of central nervous system aged $\geqslant 18$ years and diagnosed between 1995 and 2016. A total of 5714 patients were extracted from the SEER database. Similar to previous studies $\$ patients with "other infectious and parasitic diseases including HIV" as cause of death and follow-up were excluded to define a non-HIV PCNSL patient population $(n=700){ }^{22,23}$. Patients with more than one primary cancer were also excluded $(n=1026)$. Patients without pathological diagnosis and patients diagnosed at autopsy were excluded $(n=228)$. 
All methods were performed in accordance with the relevant guidelines and regulations. The datasets generated during and/or analysed during the current study are available from the corresponding author on reasonable request.

\subsection{Study variables}

According to the primary treatment the patients received, information on primary treatment were divided

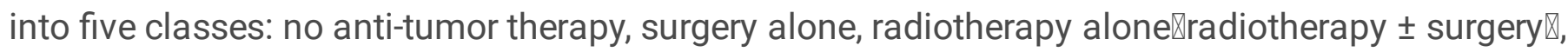

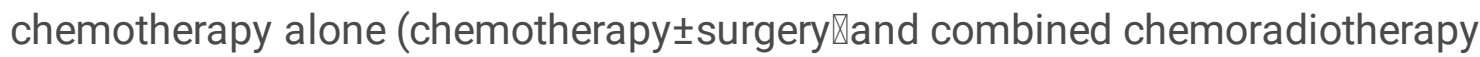
(chemoradiotherapy \pm surgery). According to the progress in treatment, the year of diagnosis was divided into three time periods. The time period- 1 is from 1995 to 2002. The time period-2 is from 2003 to 2012, a period expected to reflect more intense chemotherapy regimens, the availability of rituximab and the utilization of autologous stem-cell transplantation as consolidation strategy. The time period-3 is from 2013 to 2016, a period expected to reflect novel agents spring up. Covariates including age at diagnosis, sex, race, marital status and distribution by site in the CNS were introduced, to adjust the hazard ratio (HR). Data of Survival months, survival status, the cause of death were also collected.

\subsection{Statistical analysis}

Statistical analysis was performed using SEER stat 8.3.8 and SPSS v22.0 for Windows (SPSS Inc., Chicago, IL). Chi-square tests were used to analyze Categorical variables. Kaplan-Meier survival curves were plotted for cause-specific survival (CSS), which was defined by specifying PCNSL as the cause of death, measured from time of diagnosis of PCNSL to time of death, in months. The survival difference was compared using log-rank test. Multivariate analysis using Cox regression model was performed to identify the independent risk factors for long-term survival. Two-sided $P$ values less than 0.05 were considered to be significant.

\subsection{Ethics statement}

Ethical approval was waived by the Ethics Committee of Sun Yat-Sen University Cancer Center because preexisting data with no personal identifiers were used.

\section{Results}

\subsection{Patient characteristics}

Based on the eligibility criteria, a total of 3760 adult patients (age $\geqslant 18$ years) diagnosed with primary diffuse large B-cell lymphoma of central nervous system between 1995 and 2016 were identified in the study. Of these, 709 were diagnosed during the time period-1(1995-2002), 1989 diagnosed during the time period-2 (2003-2012) and 1062 diagnosed during the time period-3(2013-2016). Of this population, median age was 64 years (range 18-96 years). $37.1,28.5$ and $34.4 \%$ of patients were aged 18-60, 61-70 and $>70$ years, respectively. Patient characteristics of the study population stratified by the period of diagnosis are outlined and compared as shown in Table 1. Clinical features including race $(P=0.026)$, 
marital status $(P=0.004)$ and distribution by site in the CNS $(P<0.001)$ showed significant difference over the different time periods; there was no significant difference according to age $(P=0.079)$ and sex $(P=0.719)$.

\subsection{Primary treatment according to the era of diagnosis and age}

As age is an important factor in deciding treatment protocols. Information on primary treatment stratified by the period of diagnosis and age is displayed in Fig. 1. The proportion of patients received no antitumor therapy or surgery alone has not changed appreciably over time, with most of these patients aged above 60 years. There are some consistent and notable treatment changes between different age groups. The use of radiotherapy alone decreased over time, from $28.6 \%$ in the time period- 1 to $12.7 \%$ in the time period-2 and $7.8 \%$ in the time period-3. The use of radiotherapy alone all dropped substantially in different age groups, with the patients aged 18-60 years dropping most from $19.6 \%$ in the time period- 1 to $8.6 \%$ in the time period -2 and $3.9 \%$ in the time period- 3 . The application of combined chemoradiotherapy also decreased, following a wider range of use of chemotherapy alone over time. The application of combined chemoradiotherapy dropped and the use of chemotherapy widened consistently among patients in different age groups. The proportion of patients received combined chemoradiotherapy dropped from $36.2 \%$ in the time period- 1 to $23.9 \%$ in the time period- 2 and $15 \%$ in the time period- 3 . The proportion of patients received chemotherapy increased from $22.7 \%$ in the time period- 1 to $46.6 \%$ in the time period- 2 and $62.8 \%$ in the time period-3.

\subsection{Survival}

The median follow up time of the study patients was 17 months. Overall, there was a significant improvement in PCNSL cancer-specific survival (CSS), with the median CSS of all patients improving from 13 months in the time period- 1 to 19 months in the time period-2 and 41 months in the time period3. When analyzing changes in survival over time based on age, the survival increased most prominently for patients aged 18-60 years from the time period -1 to the time period-2 ( 28 months in the period time- 1 vs 71 months in the period time-2, $P=0.01$ ). Survival of patients aged above 70 years did not change from the period time- 1 to the period time-2 ( 5 months in the period time- 1 vs 5 months in the period time-2, $P=0.101)$. However, there was an increase in cancer-specific survival from the time period- 2 to the time period-3 in both younger patients (age $<70$ years) and elder patients (age $>70$ years, 5 months in the period time-2 vs 9 months in the period time-3, $P<0.001$ ). Cancer specific survival curves of adult patients with PCNSL in different age groups based on the period of diagnosis were shown in Fig. 2. The median cancer-specific survival of the study population stratified by the period of diagnosis and age are outlined in Table 2.

We analyzed the influence of age, sex, race, marital status, distribution by site in the CNS and the period of diagnosis on the relative excess risk of mortality in a multivariable model. A univariate analysis showed that age, race, marital status, distribution by site in the CNS and period of diagnosis $(P<0.05)$ were closely related to long-term cancer-specific survival. In multivariate Cox regression analysis (Table 3), diagnosed in the time period-3 was significantly and independently associated with better CSS (HR 
$0.577,95 \% \mathrm{Cl}$ 0.506-0.659). This showed no difference with the results of univariate analysis. Age and distribution by site in the CNS were also independently associated with CSS (Fig. 3, Table 3).

\section{Discussion}

PCNSL is a rare and aggressive tumor and DLBCL represents $90 \%-95 \%$ of all primary central nervous system lymphoma ${ }^{1,2}$. There are remarkable advances in the treatment of PCNSL over the last two decades $^{3,4}$. The present population-based study was conducted to detect the primary treatment and survival trends in patients with PCNSL diagnosed during 1995-2016. The results of our study indicates that both the use of radiotherapy alone and the application of combined chemoradiotherapy decreased, following more extensive use of chemotherapy alone as time went by. A significant improvement in survival was also demonstrated over time during 1995-2016.

The significantly increased cancer-specific survival from the time period- 1 to the time period- 2 is most likely bound up with the utilization of more intense chemotherapy regimens and the utilization of autologous stem-cell transplantation as consolidation strategy. As shown in our study, the use of radiotherapy alone and combined chemoradiotherapy dropped substantially with the patients aged 18-60 years dropping most and the use of chemotherapy alone increased dramatically from the time period- 1 to the time period-2. Accordingly, our results showed that the survival increased most prominently for patients aged 18-60 years who may more fit to received more intense chemotherapy or high dose-ASCT for consolidation and survival of patients aged above 70 years who may have worse functional status and greater comorbidities remained unchanged from the time period-1 to the time period-2. Although wider use of chemotherapy applicated in patients aged above 70 years, a less aggressive treatment approach may performed. Although lacking of phase III randomized controlled trials to confirm the best consolidation approach, what is clear is that the application of more intense chemotherapy and the utilization of autologous stem-cell transplantation as consolidation strategy improved survival according to our population-based study. The finding that there was no improvement in survival of patients aged above 70 years the time period- 1 to the time period-2 in our study falls in line with historical findings of previous studies based on population analysis ${ }^{24,25}$. A cohort study carried out by Mendez JS et al. shown that survival in the elderly population ( $>70$ ) has not changed from 1973 to $2013^{24}$. Van der Meulen M et al. demonstrated that improved survival was found in PCNSL up to age 70 only by a population-based study in the Netherlands, $1989-2015^{25}$.

However, there was an improvement in cancer-specific survival in the most recent period in not only younger patients ( $<70$ years) but also the elderly patients ( $>70$ years). There are many causes. One of the important reasons may be that there have been no age restrictions in several clinical trials and furthermore, trials have been conducted to target the elderly patient population (Such as PRIMAIN study) in the most recent clinical trials worldwide ${ }^{26}$. It guide clinicians making treatment decisions in this patient population. Instead, clinical trials are only applicable to young and fit patients in the past. Another possible reason is that some new agents which are also tolerated in the elderly patients springs up in the 
time period-3 as we observed that elderly patients were more likely to receive chemotherapy in the time period-3. The representative agents include an antiproliferative and immunomodulatory agent lenalidomide and an oral BTK inhibitor Ibrutinib. In a prospective multicenter study reported by Ghesquieres $\mathrm{H}$ et. al assessing the combined chemotherapy of lenalidomide plus intravenous rituximab in relapsed/refractory PCNSL, an OR rate of $67 \%$ and an overall survival of 17.7 months were reported ${ }^{18}$. Recent results from the phase II 'proof-of-concept' iLOC study investigating single-agent ibrutinib in relapse or refractory(R/R) DLBCL-PCNSL showed an OR rate of $52 \%$ and median OS of 19.2 months in 52 patients ${ }^{19}$. Monotherapy with lenalidomide or ibrutinib is also being explored as a potential consolidation or maintenance therapy, particularly in older DLBCL-PCNSL patients. It may become a clinical progress which may provide more effective treatment options and significantly improved outcome among the elderly patients with PCNSL in future.

The strength of this study is that analysis of a population-based registries SEER including a large number of DLBCL-PCNSL patients, demonstrates continuous improvement in survival during 1995-2016, which reflected developments in treatment over time. By contrast, both the use of radiotherapy alone and the application of combined chemoradiotherapy decreased in all aged groups over time. We can find that the application of radiotherapy in the treatment of PCNSL has waned over time in this large population-based study. In most recent years, and particularly after that a randomized German study (G-PCNSL-SG-1) did not show evidence of improved survival by adding radiation ${ }^{27}$, the use of whole brain radiation has been questioned. The most recent RTOG 1114 study discussing the effect of reduced-dose radiation further (RMVP/Ara-C \pm low dose WBRT) and presented orally at ASCO 2020, a better PFS was reached in the reduced-dose WBRT group compared with the chemo-alone group. However, the analyses of toxicity, especially neurotoxicity are still ongoing, the result of which might give further insight in the use of radiation in PCNSL.

Age is one of the most major prognostic factors for PCNSL. Both of the prognostic scores of Memorial Sloan Kettering Cancer Center ${ }^{28}$ and the International Extranodal Lymphoma Study Group ${ }^{29}$ include age as a prognostic factor. Survival also differed greatly between age groups and the multivariable model demonstrated an adverse effect of older age in our study. This patient population has a less radical therapeutic method, poorer performance status, and greater complication which may contribute to worse overall survival. Our study also found distribution of PCNSL by site in the CNS is another prognostic factor. Median cancer-specific survival was worse in PCNSL patients occurred in the brain parenchyma in comparison to those occurred in the CNS except brain parenchyma. Prognostic models including these factors should be developed to help in deciding the optimal therapeutic schedule in future.

It's worthy to note that our study should be considered in the context of its potential limitations. First, because of the deficiency of the SEER database, we were incapable of obtaining granular data especially the specifics on treatment (chemotherapy regimens, the dose of radiation, receive ASCT or not receive, the intent of radiotherapy and the like). Second囚information regarding prognostic factors (patient performance status, CSF protein, serum lactate dehydrogenase, and lesions within deep structures) and 
history of prior treatment is not available. Third, we were incapable of excluding every HIV-associated PCNSL.

\section{Conclusions}

Our analysis shows the use of radiotherapy in the treatment of PCNSL has waned over the study span. There was a significant improvement in survival during 1995-2016 in this large polulation-based study, which reflected developments in treatment over time. The elderly patient population also gained a significant cause-specific survival benefit in the most recent period.

\section{Declarations}

Funding: This study was supported by the Youth Funds of the Basic and Applied Basic Research Foundation of Guangdong Province, China (No. 2020A1515110089).

Conflicts of Interest: The authors declare no conflict of interest.

Authors' contributions पAll authors contributed to the study conception and design. Cui Chen, Peng Sun and Xiaoqing Sun collected the data and drafted the manuscript. Shaoyong Chen, Yu Wang and Hang Yang performed the statistical analysis. All authors commented on previous versions of the manuscript. All authors read and approved the final manuscript.

\section{References}

1. Grommes, C. \& DeAngelis, L. M. Primary CNS Lymphoma. J Clin Onco/ 35, 2410-2418 (2017).

2. Deckert, M., Brunn, A., Montesinos-Rongen, M., Terreni, M. R. \& Ponzoni, M. Primary lymphoma of the central nervous system-a diagnostic challenge. Hematol Oncol 32, 57-67 (2014).

3. Cai, Q., Fang, Y. \& Young, K. H. Primary Central Nervous System Lymphoma: Molecular Pathogenesis and Advances in Treatment. Transl Oncol 12, 523-538 (2019).

4. Holdhoff, M., Mrugala, M. M., Grommes, C., Kaley, T. J., Swinnen, L. J., Perez-Heydrich, C. \& Nayak, L. Challenges in the Treatment of Newly Diagnosed and Recurrent Primary Central Nervous System Lymphoma. J Natl Compr Canc Netw 18, 1571-1578 (2020).

5. Nelson, D. F. et al. Non-Hodgkin's lymphoma of the brain: can high dose, large volume radiation therapy improve survival? Report on a prospective trial by the Radiation Therapy Oncology Group (RTOG): RTOG 8315. Int J Radiat Oncol Biol Phys 23, 9-17 (1992).

6. O'Brien, P. C. et al. Combined-modality therapy for primary central nervous system lymphoma: longterm data from a Phase II multicenter study (Trans-Tasman Radiation Oncology Group). Int $J$ Radiat Oncol Biol Phys 64, 408-413 (2006).

7. Poortmans, P. M. et al. High-dose methotrexate-based chemotherapy followed by consolidating radiotherapy in non-AIDS-related primary central nervous system lymphoma: European Organization 
for Research and Treatment of Cancer Lymphoma Group Phase II Trial 20962. J Clin Oncol 21, 44834488 (2003).

8. Ferreri, A. J. et al. MATILDE regimen followed by radiotherapy is an active strategy against primary CNS lymphomas. Neurology 66, 1435-1438 (2006).

9. DeAngelis, L. M., Seiferheld, W., Schold, S. C., Fisher, B. \& Schultz, C. J. Combination chemotherapy and radiotherapy for primary central nervous system lymphoma: Radiation Therapy Oncology Group Study 93-10. J Clin Oncol 20, 4643-4648 (2002).

10. Illerhaus, G., Müller, F., Feuerhake, F., Schäfer, A. O., Ostertag, C. \& Finke, J. High-dose chemotherapy and autologous stem-cell transplantation without consolidating radiotherapy as first-line treatment for primary lymphoma of the central nervous system. Haematologica 93, 147-148 (2008).

11. Rubenstein, J. L. et al. Intensive chemotherapy and immunotherapy in patients with newly diagnosed primary CNS lymphoma: CALGB 50202 (Alliance 50202). J Clin Oncol31, 3061-3068 (2013).

12. Colombat, P. et al. High-dose chemotherapy with autologous stem cell transplantation as first-line therapy for primary CNS lymphoma in patients younger than 60 years: a multicenter phase II study of the GOELAMS group. Bone Marrow Transplant 38, 417-420 (2006).

13. Abrey, L. E. et al. Intensive methotrexate and cytarabine followed by high-dose chemotherapy with autologous stem-cell rescue in patients with newly diagnosed primary CNS lymphoma: an intent-totreat analysis. J Clin Oncol 21, 4151-4156 (2003).

14. Gregory, G. et al. Rituximab is associated with improved survival for aggressive B cell CNS lymphoma. Neuro Oncol 15, 1068-1073 (2013).

15. Raizer, J. J. et al. Pemetrexed in the treatment of relapsed/refractory primary central nervous system lymphoma. Cancer 118, 3743-3748 (2012).

16. Wong, E. T., Tishler, R., Barron, L. \& Wu, J. K. Immunochemotherapy with rituximab and temozolomide for central nervous system lymphomas. Cancer 101, 139-145 (2004).

17. Houillier, C. et al. Lenalidomide monotherapy as salvage treatment for recurrent primary CNS lymphoma. Neurology 84, 325-326 (2015).

18. Ghesquieres, H. et al. Lenalidomide in combination with intravenous rituximab (REVRI) in relapsed/refractory primary CNS lymphoma or primary intraocular lymphoma: a multicenter prospective 'proof of concept' phase II study of the French Oculo-Cerebral lymphoma (LOC) Network and the Lymphoma Study Association (LYSA)t. Ann Onco/ 30, 621-628 (2019).

19. Soussain, C. et al. Ibrutinib monotherapy for relapse or refractory primary CNS lymphoma and primary vitreoretinal lymphoma: Final analysis of the phase II 'proof-of-concept' iLOC study by the Lymphoma study association (LYSA) and the French oculo-cerebral lymphoma (LOC) network. Eur J Cancer 117, 121-130 (2019).

20. Nayak, L. et al. PD-1 blockade with nivolumab in relapsed/refractory primary central nervous system and testicular lymphoma. Blood 129, 3071-3073 (2017).

21. Noone, A. M., Cronin, K. A., Altekruse, S. F., Howlader, N., Lewis, D. R., Petkov, V. I. \& Penberthy, L. Cancer Incidence and Survival Trends by Subtype Using Data from the Surveillance Epidemiology 
and End Results Program, 1992-2013. Cancer Epidemiol Biomarkers Prev 26, 632-641 (2017).

22. Norden, A. D., Drappatz, J., Wen, P. Y. \& Claus, E. B. Survival among patients with primary central nervous system lymphoma, 1973-2004. J Neurooncol 101, 487-493 (2011).

23. Panageas, K. S., Elkin, E. B., DeAngelis, L. M., Ben-Porat, L. \& Abrey, L. E. Trends in survival from primary central nervous system lymphoma, 1975-1999: a population-based analysis. Cancer 104, 2466-2472 (2005).

24. Mendez, J. S., Ostrom, Q. T., Gittleman, H., Kruchko, C., DeAngelis, L. M., Barnholtz-Sloan, J. S. \& Grommes, $\mathrm{C}$. The elderly left behind-changes in survival trends of primary central nervous system lymphoma over the past 4 decades. Neuro Oncol 20, 687-694 (2018).

25. van der Meulen, M., Dinmohamed, A. G., Visser, O., Doorduijn, J. K. \& Bromberg, J. Improved survival in primary central nervous system lymphoma up to age 70 only: a population-based study on incidence, primary treatment and survival in the Netherlands, 1989-2015. Leukemia 31, 1822-1825 (2017).

26. Fritsch, K. et al. High-dose methotrexate-based immuno-chemotherapy for elderly primary CNS lymphoma patients (PRIMAIN study). Leukemia 31, 846-852 (2017).

27. Korfel, A. et al. Randomized phase III study of whole-brain radiotherapy for primary CNS lymphoma. Neurology 84, 1242-1248 (2015).

28. Abrey, L. E. et al. Primary central nervous system lymphoma: the Memorial Sloan-Kettering Cancer Center prognostic model. J Clin Oncol 24, 5711-5715 (2006).

29. Ferreri, A. J. et al. Prognostic scoring system for primary CNS lymphomas: the International Extranodal Lymphoma Study Group experience. J Clin Oncol 21, 266-272 (2003).

\section{Tables}

Table 1. Patient Characteristics and Comparison of the study population stratified by the period of diagnosis 


\begin{tabular}{|llllll|}
\hline & & period-1 & period-2 & period-3 & \\
\hline Variable & Total & $1995-2002$ & $2003-2012$ & $2013-2016$ & P-value \\
\hline Number of patients (\%) & 3760 & 709 & 1989 & 1062 & \\
\hline Age (years) & & & & & 0.079 \\
\hline Median & 64 & 65 & 64 & 66 & \\
\hline 18-60 & $1394(37.1)$ & $260(36.7)$ & $775(39)$ & $359(33.8)$ & \\
\hline 60-70 & $1073(28.5)$ & $199(28.1)$ & $558(28.1)$ & $316(29.8)$ & \\
\hline$>70$ & $1293(34.4)$ & $250(35.3)$ & $656(33)$ & $387(36.4)$ & \\
\hline Sex (\%) & & & & & 0.719 \\
\hline Female & $1874(49.1)$ & $351(49.5)$ & $965(48.5)$ & $531(50)$ & \\
\hline Male & $1913(50.9)$ & $358(50.5)$ & $1024(51.5)$ & $531(50)$ & \\
\hline Race(\%) & & & & & $0.026^{*}$ \\
\hline White & $3076(81.8)$ & $602(84.9)$ & $1634(82.2)$ & $840(79.1)$ & \\
\hline Black & $189(5)$ & $31(4.4)$ & $102(5.1)$ & $56(5.3)$ & \\
\hline Other & $349(9.3)$ & $71(10)$ & $154(7.7)$ & $124(11.7)$ & \\
\hline Marital status & $395(13.2)$ & $76(10.7)$ & $253(12.7)$ & $166(15.6)$ & \\
\hline Married & & & & & $0.004^{*}$ \\
\hline Widowed & $30(0.8)$ & $9(1.3)$ & $16(0.8)$ & $5(0.5)$ & \\
\hline Other & & & & & \\
\hline Distribution by site & $2274(60.5)$ & $424(59.8)$ & $1199(60.3)$ & $651(61.3)$ & \\
\hline Brain parenchyma & $422(11.2)$ & $102(14.4)$ & $228(11.5)$ & $92(8.7)$ & \\
\hline Leptomeninges & $319(30)$ & $183(25.8)$ & $562(28.3)$ & $319(30)$ & \\
\hline Spine cord,cranial nerves & & & & & \\
\hline CNS not otherwise specified & & & & \\
\hline
\end{tabular}

$\star P<0.05$

Table 2. Median Cancer-specific Survival Based on Age, months 


\begin{tabular}{|lllll|}
\hline Time of diagnosis & all patients & $18-60$ years & $61-70$ years & $>70$ years \\
\hline Period-1(1995-2002) & 13 & 28 & 16 & 5 \\
\hline Period-2(2003-2012) & 19 & 71 & 21 & 5 \\
\hline Period-3(2013-2016) & 41 & Not Reached & 39 & 9 \\
\hline$P$-value for trend & $<0.001^{*}$ & $<0.001^{*}$ & $0.004^{*}$ & $<0.001^{\star}$ \\
\hline
\end{tabular}

$\star P<0.05$

Table 3. Multivariable analysis 


\begin{tabular}{|c|c|c|c|c|c|}
\hline \multirow[t]{2}{*}{ Variable } & \multicolumn{2}{|c|}{ Univariate analysis } & \multicolumn{2}{|c|}{ Multivariate analysis } & \multirow[b]{2}{*}{$P$-value } \\
\hline & $P$-value & $\mathrm{HR}$ & $95 \% \mathrm{Cl}$ & & \\
\hline Age (years) & $<0.001^{\star *}$ & & & & $<0.001^{*}$ \\
\hline $18-60$ & & 1 (Ref) & & & \\
\hline $61-70$ & & 1.552 & 1.389 & 1.734 & $<0.001^{*}$ \\
\hline$>70$ & & 2.578 & 2.325 & 2.858 & $<0.001^{*}$ \\
\hline Sex & 0.362 & & & & \\
\hline \multicolumn{6}{|l|}{ Female } \\
\hline \multicolumn{6}{|l|}{ Male } \\
\hline Race & 0.007 & & & & 0.123 \\
\hline White & & 1 (Ref) & & & \\
\hline Black & & 0.993 & 0.805 & 1.225 & 0.946 \\
\hline Other & & 0.874 & 0.767 & 0.994 & $0.041 *$ \\
\hline Marital status & 0.851 & & & & \\
\hline \multicolumn{6}{|l|}{ Married } \\
\hline \multicolumn{6}{|l|}{ Widowed } \\
\hline \multicolumn{6}{|l|}{ Other } \\
\hline Period of diagnosis & $<0.001 *$ & & & & $<0.001^{\star}$ \\
\hline $1995-2002$ & & 1 (Ref) & & & \\
\hline $2002-2012$ & & 0.827 & 0.748 & 0.916 & $<0.001^{\star}$ \\
\hline 2013-2016 & & 0.577 & 0.506 & 0.659 & $<0.001^{\star}$ \\
\hline Distribution & $<0.001^{*}$ & & & & $<0.001^{*}$ \\
\hline Brain parenchyma & & 1 (Ref) & & & \\
\hline Leptomeninges & & 0.305 & 0.152 & 0.612 & $0.001 *$ \\
\hline Spine cord,cranial nerves & & 0.365 & 0.272 & 0.489 & $<0.001^{*}$ \\
\hline CNS not otherwise specified & & 0.783 & 0.672 & 0.913 & $0.002^{\star}$ \\
\hline
\end{tabular}

${ }^{\star} P<0.05$

Figures 
A

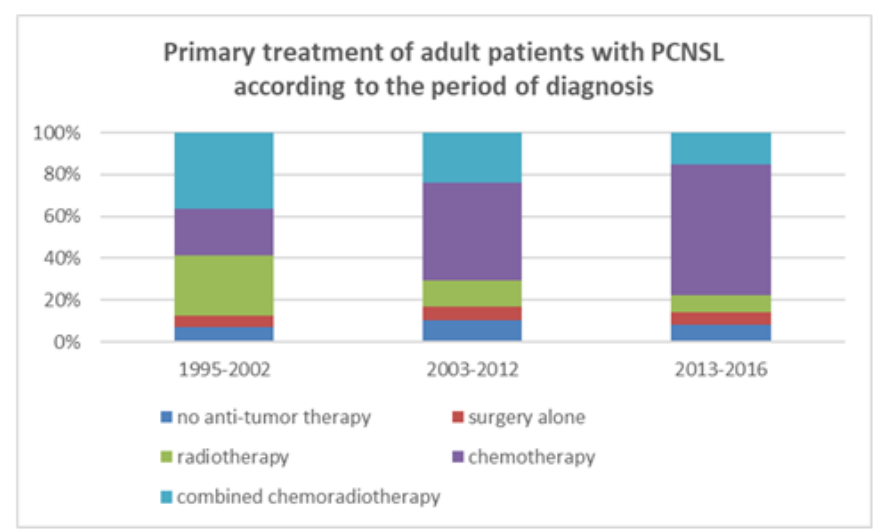

C

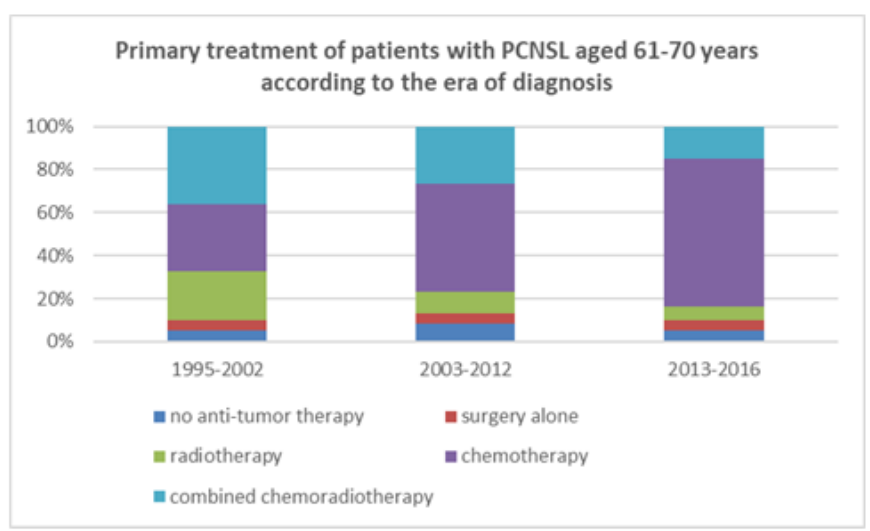

B

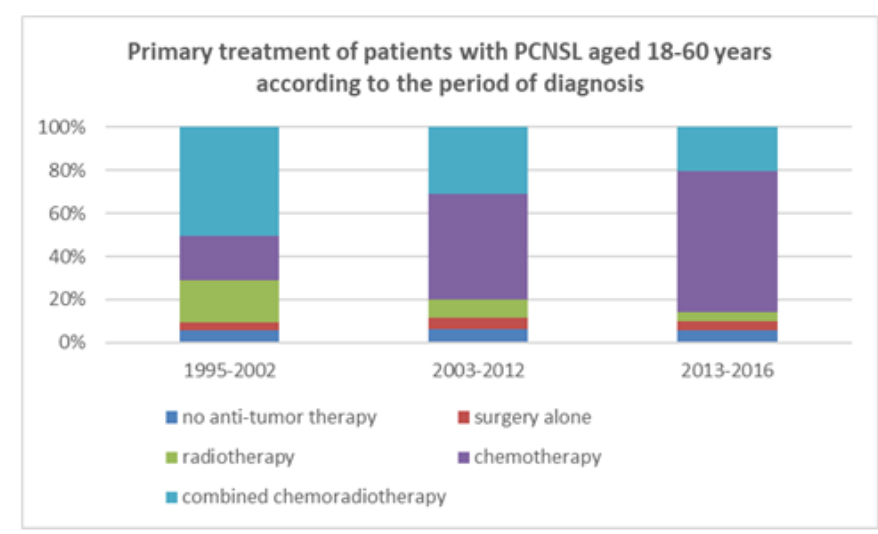

D

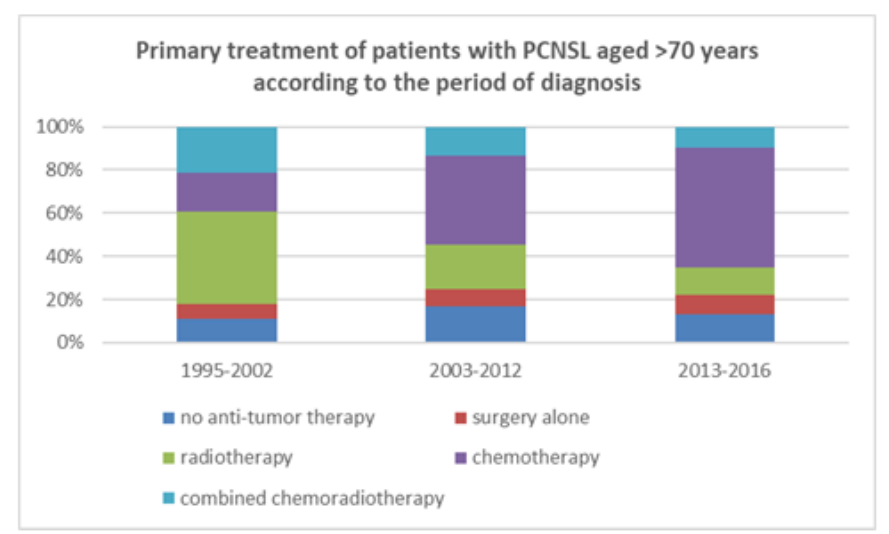

\section{Figure 1}

Primary treatment of adult patients with PCNSL according to the period of diagnosis (a) All patients (b) Patients aged 18-60 years \c囚Patients aged 61-70 years (d) Patients aged $>70$ years 
A

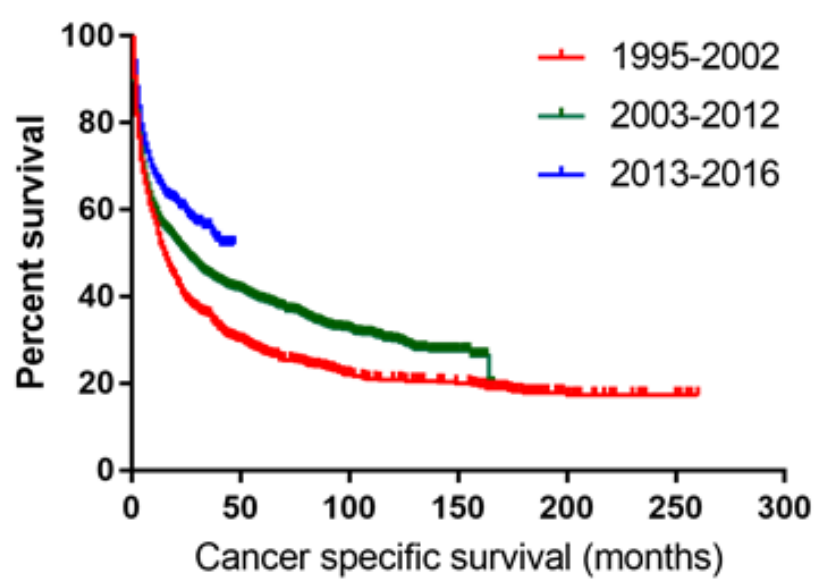

C

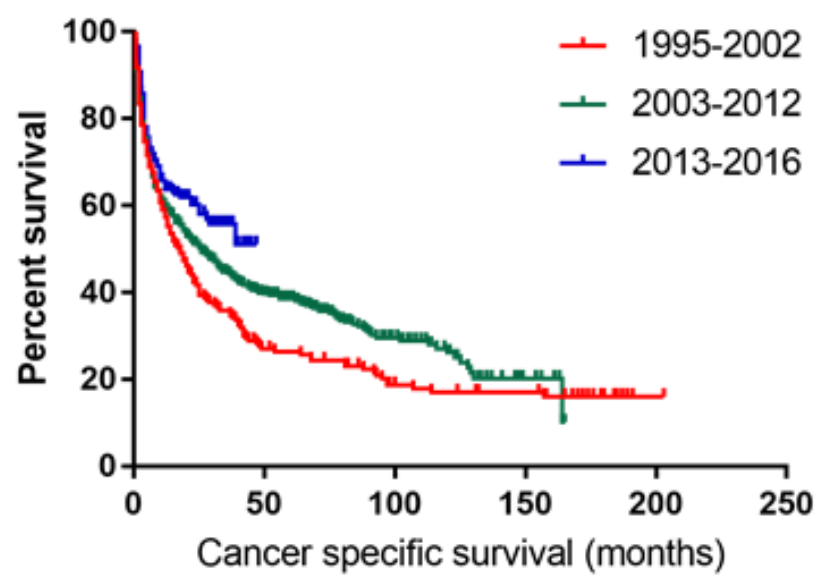

B
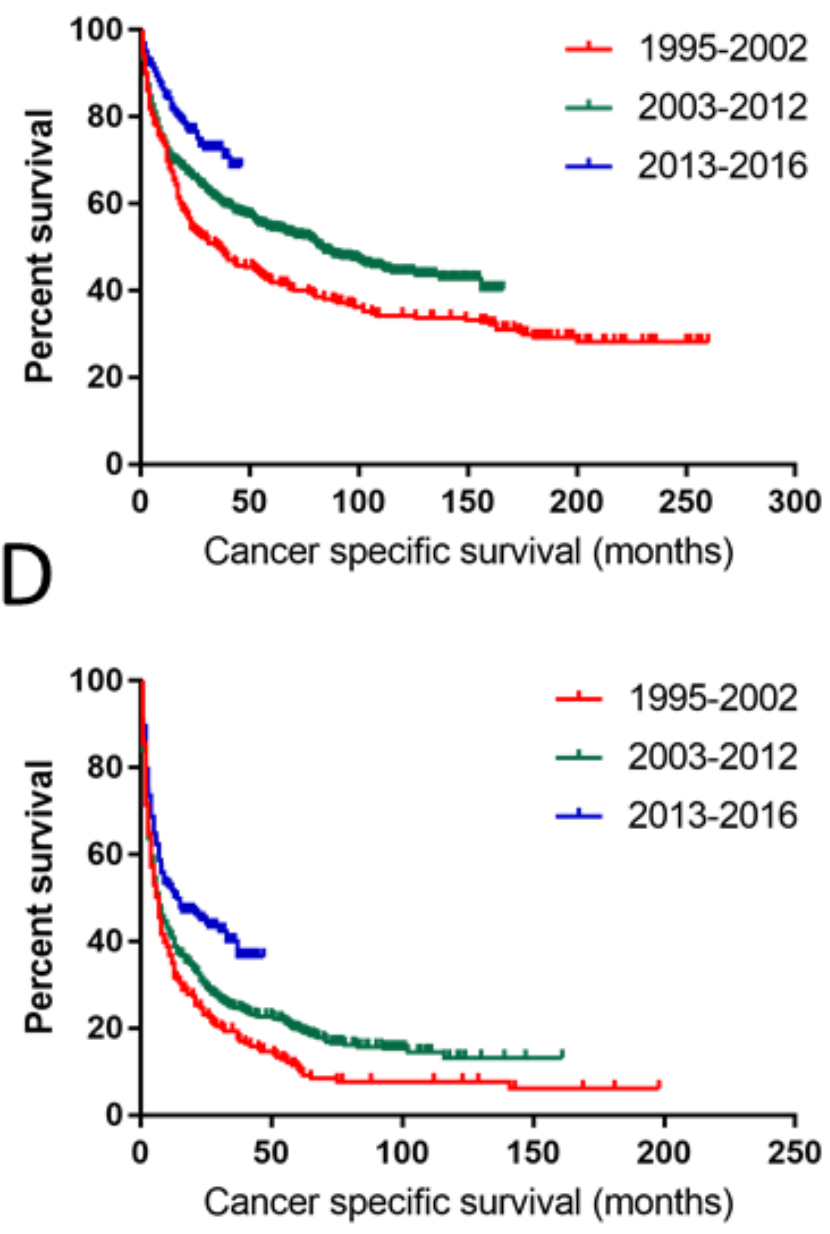

Figure 2

Cancer specific survival curves of adult patients with PCNSL based on the period of diagnosis. (a) All patients (b) Patients aged 18-60 years (c) Patients aged 61-70 years (d) Patients aged $>70$ years 

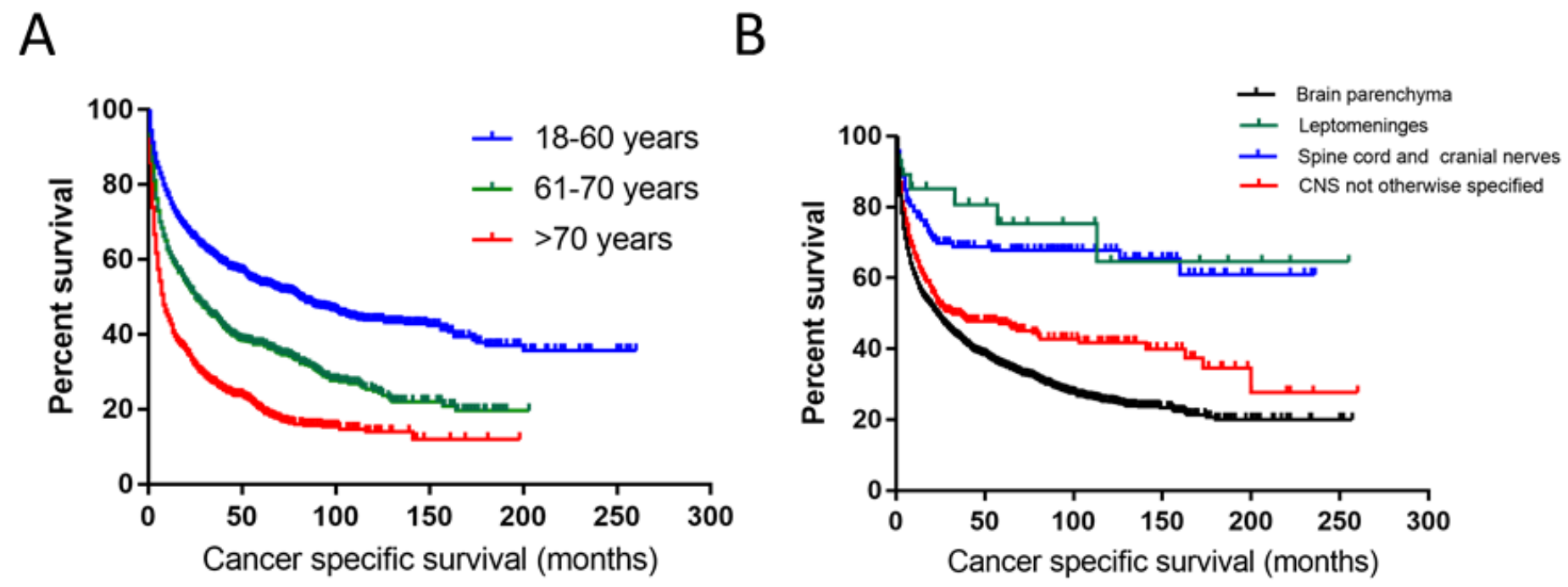

Figure 3

Cancer specific survival curves of all PCNSL patients based on (a) age and (b) distribution by site in the CNS

\section{Supplementary Files}

This is a list of supplementary files associated with this preprint. Click to download.

- Data.sav 